\title{
Comparison of DFT Methods for Molecular Orbital Eigenvalue Calculations
}

\author{
Gang Zhang and Charles B. Musgrave* \\ Department of Chemical Engineering \\ Stanford University, Stanford, CA 94305
}

*Author for correspondence. Email: chasm@stanford.edu 

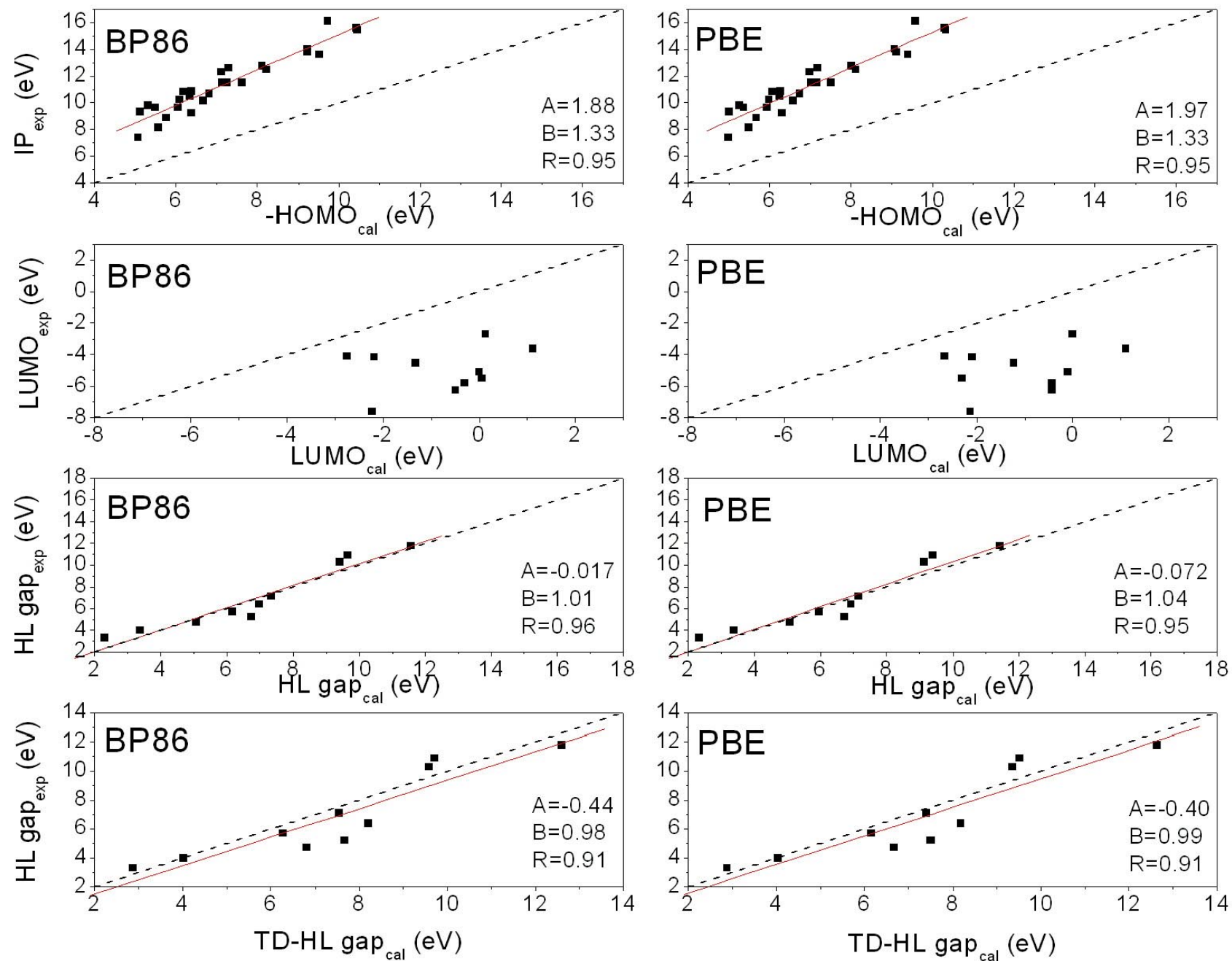

Figure 1. The calculated values versus experimental values for HOMO eigenvalues, LUMO eigenvalues, HOMO-LUMO gaps and TD-DFT gaps for BP86 and PBE functionals. The dashed line indicates an ideal 1.0 correlation between experimental and calculated values. 
Table1. Corrected HOMO eigenvalues (in eV), using the correction formula in Equation 1 with values given in Figure 1.

\begin{tabular}{|c|c|c|c|c|c|c|c|c|c|c|c|c|}
\hline & SVWN & BLYP & BP86 & BPW91 & PW91 & PBE & B3LYP & KMLYP & BH\&HLYP & O3LYP & B1B95 & EXP \\
\hline $\mathrm{HCO}$ & -8.34 & -8.72 & -8.65 & -8.67 & -8.64 & -8.63 & -8.90 & -9.17 & -9.28 & -8.75 & -8.89 & -9.31 \\
\hline $\mathrm{CO}_{2}$ & -14.29 & -14.06 & -14.11 & -14.11 & -14.12 & -14.12 & -14.03 & -13.96 & -13.91 & -14.07 & -14.05 & -13.78 \\
\hline $\mathrm{C}_{2} \mathrm{H}_{2}$ & -11.61 & -11.42 & -11.50 & -11.49 & -11.49 & -11.51 & -11.27 & -11.09 & -11.06 & -11.34 & -11.29 & -11.49 \\
\hline $\mathrm{H}_{2} \mathrm{CO}$ & -10.28 & -10.40 & -10.34 & -10.35 & -10.34 & -10.32 & -10.63 & -10.93 & -10.93 & -10.49 & -10.67 & -10.88 \\
\hline $\mathrm{CH}_{4}$ & -14.37 & -14.50 & -14.49 & -14.50 & -14.50 & -14.50 & -14.34 & -14.00 & -14.07 & -14.44 & -14.29 & -13.60 \\
\hline $\mathrm{H}_{2} \mathrm{CCO}$ & -9.98 & -9.87 & -9.89 & -9.89 & -9.88 & -9.88 & -9.78 & -9.72 & -9.70 & -9.77 & -9.78 & -9.64 \\
\hline $\mathrm{Cl}_{2}$ & -11.91 & -11.99 & -11.99 & -11.98 & -11.96 & -11.99 & -11.87 & -11.64 & -11.70 & -11.91 & -11.81 & -11.49 \\
\hline $\mathrm{CO}$ & -13.99 & -14.10 & -14.09 & -14.08 & -14.07 & -14.06 & -14.09 & -13.94 & -13.99 & -14.07 & -14.05 & -14.01 \\
\hline $\mathrm{H}_{2}$ & -15.31 & -15.81 & -15.72 & -15.76 & -15.71 & -15.72 & -15.60 & -15.03 & -15.24 & -15.72 & -15.50 & -15.43 \\
\hline $\mathrm{N}_{2}$ & -15.72 & -15.71 & -15.70 & -15.67 & -15.69 & -15.68 & -15.83 & -15.88 & -15.83 & -15.73 & -15.81 & -15.58 \\
\hline $\mathrm{O}_{2}$ & -11.12 & -11.50 & -11.31 & -11.34 & -11.32 & -11.28 & -11.98 & -12.60 & -12.63 & -11.67 & -12.01 & -12.30 \\
\hline $\mathrm{CH}_{3} \mathrm{NH}_{2}$ & -8.93 & -9.16 & -9.14 & -9.14 & -9.10 & -9.11 & -9.33 & -9.60 & -9.65 & -9.26 & -9.39 & -9.65 \\
\hline $\mathrm{CH}_{3} \mathrm{CHO}$ & -9.92 & -10.01 & -9.96 & -9.94 & -9.95 & -9.93 & -10.24 & -10.58 & -10.56 & -10.09 & -10.28 & -10.24 \\
\hline $\mathrm{C}_{4} \mathrm{H}_{4} \mathrm{O}$ & -9.65 & -9.49 & -9.52 & -9.52 & -9.53 & -9.52 & -9.26 & -9.03 & -9.03 & -9.29 & -9.26 & -8.90 \\
\hline $\mathrm{HF}$ & -14.83 & -14.81 & -14.76 & -14.75 & -14.78 & -14.76 & -15.27 & -15.83 & -15.70 & -15.10 & -15.42 & -16.12 \\
\hline $\mathrm{C}_{2} \mathrm{H}_{4}$ & -11.09 & -10.84 & -10.91 & -10.92 & -10.93 & -10.95 & -10.62 & -10.37 & -10.34 & -10.72 & -10.66 & -10.68 \\
\hline $\mathrm{C}_{4} \mathrm{H}_{2}$ & -10.84 & -10.64 & -10.71 & -10.72 & -10.71 & -10.73 & -10.41 & -10.19 & -10.17 & -10.50 & -10.41 & -10.17 \\
\hline $\mathrm{C}_{6} \mathrm{H}_{6}$ & -10.55 & -10.24 & -10.34 & -10.35 & -10.36 & -10.37 & -9.92 & -9.56 & -9.52 & -10.20 & -9.80 & -9.25 \\
\hline $\mathrm{C}_{10} \mathrm{H}_{8}$ & -9.42 & -9.16 & -9.26 & -9.25 & -9.26 & -9.27 & -8.81 & -8.43 & -8.43 & -9.06 & -8.69 & -8.14 \\
\hline $\mathrm{C}_{14} \mathrm{H}_{10}$ & -8.75 & -8.52 & -8.59 & -8.59 & -8.60 & -8.61 & -8.12 & -7.72 & -7.73 & -8.35 & -7.99 & -7.40 \\
\hline $\mathrm{H}_{2} \mathrm{O}$ & -11.57 & -11.59 & -11.55 & -11.54 & -11.56 & -11.53 & -11.98 & -12.50 & -12.42 & -11.81 & -12.08 & -12.62 \\
\hline $\mathrm{H}_{2} \mathrm{~S}$ & -10.24 & -10.24 & -10.28 & -10.29 & -10.27 & -10.29 & -10.17 & -10.08 & -10.10 & -10.25 & -10.18 & -10.50 \\
\hline $\mathrm{HCl}$ & -12.62 & -12.57 & -12.64 & -12.63 & -12.62 & -12.65 & -12.49 & -12.35 & -12.35 & -12.60 & -12.51 & -12.74 \\
\hline $\mathrm{NaCl}$ & -9.06 & -8.97 & -8.93 & -8.90 & -8.97 & -8.97 & -8.99 & -9.18 & -9.14 & -8.94 & -8.98 & -9.80 \\
\hline $\mathrm{NH}_{3}$ & -9.91 & -10.07 & -10.06 & -10.06 & -10.04 & -10.05 & -10.25 & -10.53 & -10.53 & -10.21 & -10.34 & -10.82 \\
\hline $\mathrm{HCOOH}$ & -11.37 & -11.38 & -11.32 & -11.32 & -11.34 & -11.32 & -11.56 & -11.86 & -11.81 & -11.42 & -11.61 & -11.50 \\
\hline $\mathrm{SO}_{2}$ & -12.88 & -12.78 & -12.78 & -12.77 & -12.81 & -12.79 & -12.79 & -12.75 & -12.73 & -12.77 & -12.77 & -12.50 \\
\hline $\begin{array}{l}\text { Largest } \\
\text { error }\end{array}$ & 1.35 & 1.31 & 1.36 & 1.37 & 1.34 & 1.36 & 0.85 & 0.62 & 0.66 & 1.02 & 0.82 & \\
\hline $\begin{array}{l}\text { Average } \\
\text { error }\end{array}$ & 0.62 & 0.53 & 0.56 & 0.56 & 0.56 & 0.57 & 0.37 & 0.24 & 0.25 & 0.45 & 0.33 & \\
\hline
\end{tabular}


Table2. Corrected HOMO-LUMO gaps (in eV), using the correction formula in Equation 1 with values given in Figure 3.

\begin{tabular}{|l|l|l|l|l|l|l|l|l|l|l|l|l|}
\hline & SVWN & BLYP & BP86 & BPW91 & PW91 & PBE & B3LYP & KMLYP & BH\&HLYP & O3LYP & B1B95 & EXP \\
\hline $\mathrm{C}_{2} \mathrm{H}_{2}$ & 6.94 & 6.94 & 6.83 & 6.85 & 6.95 & 6.92 & 6.91 & 6.56 & 6.46 & 6.94 & 6.91 & 5.23 \\
\hline $\mathrm{CH}_{4}$ & 9.86 & 9.68 & 9.77 & 9.74 & 9.66 & 9.69 & 9.22 & 8.62 & 8.67 & 9.21 & 9.03 & 10.9 \\
\hline $\mathrm{CO}$ & 6.99 & 7.26 & 7.08 & 7.06 & 7.19 & 7.14 & 7.70 & 8.17 & 8.22 & 7.60 & 7.75 & 6.4 \\
\hline $\mathrm{H}_{2}$ & 11.55 & 11.88 & 11.72 & 11.75 & 11.82 & 11.80 & 11.40 & 10.65 & 10.92 & 11.65 & 11.12 & 11.8 \\
\hline $\mathrm{HF}$ & 9.66 & 9.34 & 9.53 & 9.48 & 9.42 & 9.44 & 9.74 & 10.26 & 10.12 & 9.55 & 9.90 & 10.3 \\
\hline $\mathrm{C}_{6} \mathrm{H}_{6}$ & 5.23 & 5.22 & 5.13 & 5.13 & 5.24 & 5.21 & 5.11 & 5.06 & 5.06 & 5.27 & 4.99 & 4.72 \\
\hline $\mathrm{C}_{10} \mathrm{H}_{8}$ & 3.40 & 3.47 & 3.40 & 3.41 & 3.45 & 3.44 & 3.39 & 3.44 & 3.46 & 3.45 & 3.35 & 3.99 \\
\hline $\mathrm{C}_{14} \mathrm{H}_{10}$ & 2.26 & 2.38 & 2.33 & 2.33 & 2.34 & 2.34 & 2.28 & 2.35 & 2.37 & 2.29 & 2.29 & 3.31 \\
\hline $\mathrm{H}_{2} \mathrm{O}$ & 7.44 & 7.27 & 7.44 & 7.46 & 7.35 & 7.37 & 7.63 & 8.14 & 8.03 & 7.53 & 7.86 & 7.1 \\
\hline $\mathrm{NH}_{3}$ & 6.14 & 6.03 & 6.24 & 6.26 & 6.04 & 6.12 & 6.09 & 6.22 & 6.16 & 5.98 & 6.27 & 5.72 \\
\hline $\begin{array}{l}\text { Largest } \\
\text { error }\end{array}$ & 1.71 & 1.71 & 1.60 & 1.62 & 1.72 & 1.69 & 1.68 & 2.28 & 2.23 & 1.71 & 1.87 & \\
\hline $\begin{array}{l}\text { Average } \\
\text { error }\end{array}$ & 0.71 & 0.73 & 0.71 & 0.72 & 0.73 & 0.72 & 0.85 & 1.00 & 0.95 & 0.83 & 0.92 & \\
\hline
\end{tabular}


Table3. Corrected TD-DFT HOMO-LUMO gaps (in eV), using the correction formula in Equation 1 with values given in Figure 4.

\begin{tabular}{|l|l|l|l|l|l|l|l|l|l|l|l|l|}
\hline & SVWN & BLYP & BP86 & BPW91 & PW91 & PBE & B3LYP & KMLYP & BH\&HLYP & O3LYP & B1B95 & EXP \\
\hline $\mathrm{C}_{2} \mathrm{H}_{2}$ & 7.28 & 6.51 & 7.08 & 7.03 & 6.97 & 7.01 & 6.31 & 7.22 & 6.88 & 6.71 & 6.99 & 5.23 \\
\hline $\mathrm{CH}_{4}$ & 9.08 & 9.17 & 9.09 & 9.05 & 8.94 & 8.99 & 9.37 & 9.45 & 9.28 & 8.90 & 9.18 & 10.9 \\
\hline $\mathrm{CO}$ & 7.59 & 7.93 & 7.60 & 7.58 & 7.72 & 7.68 & 7.83 & 7.39 & 7.40 & 7.74 & 7.43 & 6.4 \\
\hline $\mathrm{H}_{2}$ & 11.74 & 12.45 & 11.93 & 12.00 & 12.16 & 12.08 & 12.27 & 11.65 & 11.89 & 12.27 & 11.90 & 11.8 \\
\hline $\mathrm{HF}$ & 9.01 & 8.93 & 8.96 & 8.91 & 8.80 & 8.84 & 9.30 & 9.77 & 9.47 & 8.91 & 9.27 & 10.3 \\
\hline $\mathrm{C}_{6} \mathrm{H}_{6}$ & 6.35 & 5.07 & 6.23 & 6.23 & 6.11 & 6.20 & 4.82 & 4.56 & 5.91 & 6.06 & 6.06 & 4.72 \\
\hline $\mathrm{C}_{10} \mathrm{H}_{8}$ & 3.52 & 3.90 & 3.50 & 3.51 & 3.63 & 3.59 & 3.80 & 3.63 & 3.45 & 3.68 & 3.48 & 3.99 \\
\hline $\mathrm{C}_{14} \mathrm{H}_{10}$ & 2.32 & 2.72 & 2.37 & 2.38 & 2.48 & 2.44 & 2.66 & 2.53 & 2.35 & 2.49 & 2.32 & 3.31 \\
\hline $\mathrm{H}_{2} \mathrm{O}$ & 6.93 & 6.99 & 6.96 & 6.98 & 6.91 & 6.92 & 7.18 & 7.32 & 7.08 & 6.97 & 7.06 & 7.1 \\
\hline $\mathrm{NH}_{3}$ & 5.59 & 5.76 & 5.70 & 5.75 & 5.70 & 5.68 & 5.89 & 5.92 & 5.70 & 5.69 & 5.73 & 5.72 \\
\hline $\begin{array}{l}\text { Largest } \\
\text { error }\end{array}$ & 2.08 & 1.73 & 1.88 & 1.85 & 1.96 & 1.91 & 1.53 & 2.02 & 1.68 & 2.00 & 1.79 & \\
\hline $\begin{array}{l}\text { Average } \\
\text { error }\end{array}$ & 0.98 & 0.78 & 0.94 & 0.95 & 0.97 & 0.97 & 0.68 & 0.69 & 0.79 & 0.93 & 0.86 & \\
\hline
\end{tabular}

